\title{
Increasing the energy efficiency of OFDM systems using differential signal conversion
}

\author{
G.S. Voronkov ${ }^{1}$, I.V. Kuznetsov ${ }^{1}$, A.Kh. Sultanov ${ }^{1}$ \\ ${ }^{1}$ Ufa State Aviational Technical University, 12, K. Marx St., 450000, Ufa, Russia
}

\begin{abstract}
A method for improving the energy efficiency of OFDM systems based on differential transformation and extrapolation is considered. The possible structure of the extrapolator is analyzed, the implementation of an extrapolator based on the Kalman - Bucy filter and the Wiener filter is considered. The results of carried out simulation confirming the effectiveness of the proposed scheme are given.
\end{abstract}

Keywords: OFDM; dynamic range; compression; extrapolation; energy efficiency; differential method

\section{Introduction}

One of the main directions of the development of telecommunication systems nowadays is the data rate increase. Quadrature modulation and orthogonal frequency division multiplexing are used to solve this problem. Mobile networks of 4th and 5th generation [1,2] and modern satellite communications [3] can be referred as the examples of this. But data rate increase requires signal-to-noise ratio increase while keeping channel parameters unchanged. This cause transmitting power increase. The power increase, in turn, makes the process of microwave end amplifiers development and leads the devices power consumption increase which decreases their recharge interval. Various methods are currently used to reduce the radiated power. Base station (BTS) manages the mobile terminal (MT) power using control channel in 2d generation mobile networks (GSM, DCS). BTS measures the received from MT signal strength during the communication session and adjusts MT radiated power. This is an iterative process, MT power is being reduced gradually until optimal threshold level [4]. When degradation of communication quality is detected, BTS increases MT radiated power gradually in the same way until its maximum. This method obviously requires dedicates control channel. Using automatic control theory terms, it is feedback path control.

Another power control procedure is used in 3d and 4th (UMTS, LTE) generation mobile networks. Mobile terminals in these networks transmit and receive signals in the same frequency channel in one BTS cell sector, so MT output power is varied not from its maximum value to the minimum, but on the contrary - from the minimum to the optimal value that guarantees the predefined communication quality (to reduce electromagnetic disturbance for the other MT). Output power is being changed according the base station commands [1,5]. It is important to note that the method described doesn't reduce transmitter maximum output power but only adjusts its according to the signal propagation conditions to provide predefined communication quality.

Some methods allow maximum output power decrease, that provides recharge interval and life utility [6] growth. For example, multi-dimensional signal constellation method is known. It allows to reduce output power by $1 \mathrm{~dB}$ [7], but requires quadrature modulators of consisting telecommunication system replacing, because it changes constellation forming algorithm. So, this method increases the complexity of devices.

It is suggested to use differential transformation of OFDM signals to reduce a transmitter output power without communication quality decrease and device complexity increase. Differential transformation means OFDM signal dynamic range decrease by extrapolating of its values. In this case, the extrapolator transfer function must be synthesized considering the signal properties and the channel noise. Differential transformation allows to reduce OFDM band signal power while keeping communication system noise stability unchanged, that is considered in this paper.

\section{The extrapolation method}

Two methods of differential transformation can be offered in general: using input control ("input method") or output control ("output method"). OFDM signal generating and receiving general scheme is given in Fig. 1.

The extrapolator parameters should be known on the receiving side to receive transformed signal correctly. These parameters are suggested to be transferred using secondary communication channel to reduce the calculation amount. If extrapolator parameters are changed slow compared with signal, secondary channel may be considered as a lossless channel, so transmitter and receiver extrapolators are equal.

In the differential transformation "input method" schema design Kalman - Bucy filter can be used as the extrapolator. Such schema advantages are:

1) considering channel moise directly in the model;

2) both stationary and non-stationary signals processing;

3) solving the problem in digital form.

The schema disadvantages are:

1) significant prediction error in the initial stages of signal observation;

2) problem of stability guaranteeing. 
Image Processing, Geoinformation Technology and Information Security / G.S. Voronkov, I.V. Kuznetsov, A.Kh. Sultanov

Another disadvantage is watch and state equations system solving necessity, which is somewhat complicated.

Taking into consider these disadvantages, it is suggested to use the schema of differential transformation based on "output method" and to synthesize extrapolator transfer function based on Wiener-Hopf equation solution. It is also suggested to reduce the number of schema elements using one common extrapolator with coordination function to process both signals of in-phase and quadrature channels as it is shown in [5]. The calculation model used for this case shown in Fig. 2.

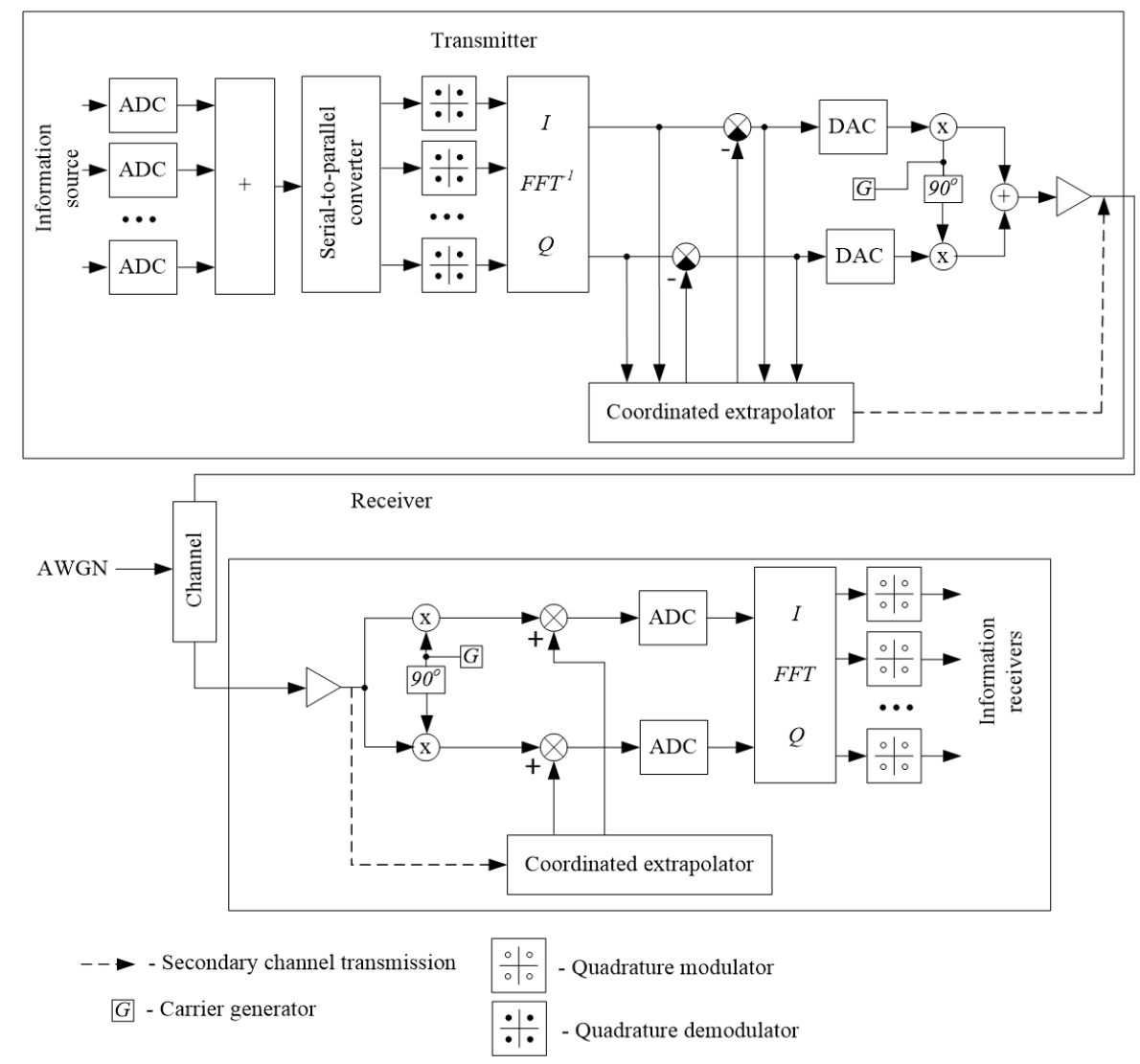

Fig. 1. OFDM signal with differential tranformation generating and receiving general.

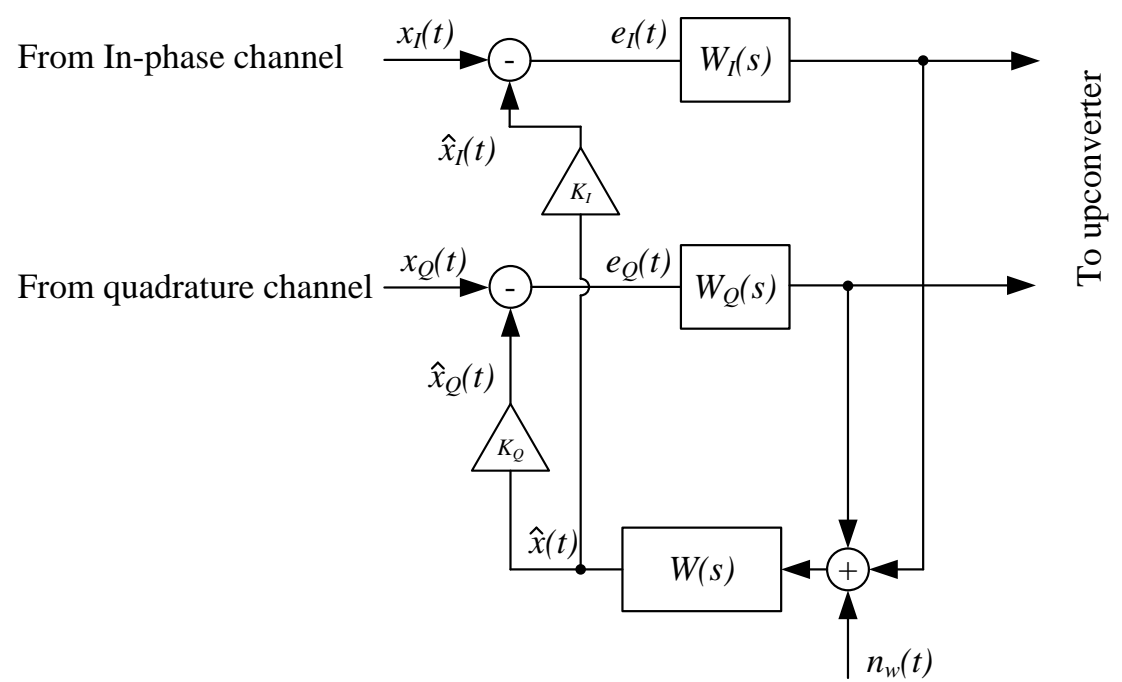

Fig. 2. OFDM-signal transmitter with coordinated extrapolator calculation model

Functions designated as $x_{I}(t), x_{Q}(t)$ are OFDM band signal in-phase and quadrature components on the invers Fourier transform unit output, $\hat{x}_{I}(t), \hat{x}_{Q}(t)$ - extrapolated values of the corresponding functions, $e_{I}(t), e_{Q}(t)$ - difference signals. Channel noise $n_{w}(t)$, which spectral power density (SPD) $\Phi_{N N}$ is assumed to be known, is also taken in consideration in the proposed extrapolator schema transfer function $W(s)(s$ - complex Laplace variable) synthesis process. Transfer functions designated as $W_{I}(s)$ and $W_{Q}(s)$ are introduced to describe the transformation and delay processes in the in-phase and quadrature channels and are also known. The transfer function synthesis is described in $[4,5]$. In general, the extrapolator transfer function can be represented in the form below: 


$$
\begin{aligned}
& \text { Image Processing, Geoinformation Technology and Information Security / G.S. Voronkov, I.V. Kuznetsov, A.Kh. Sultanov } \\
& W(s)=\frac{1}{\Phi_{z z}^{+}(s)}\left[\frac{\Phi_{z x}(s)}{\Phi_{z z}^{-}(s)}\right]_{+}
\end{aligned}
$$

where $\Phi(s)$ - cross-spectral density of signals designated in the index;

$x(t)$ - OFDM band signal in-phase or quadrature (according to the index) component;

$z(t)$ - OFDM band signal in-phase or quadrature component and channel noise mixed signal, $z(t)=x(t)+n_{w}(t)$.

The solution for the special case of PSD that represented in the form below was obtained in [5]:

$$
\begin{gathered}
\Phi_{x x}(s)=\frac{1}{a_{1}^{2}\left(\alpha^{2}-s^{2}\right)}, \\
\Phi_{n n}(s)=\frac{1}{a_{2}^{2}} .
\end{gathered}
$$

Extrapolator transfer function in the case described is:

$$
W(s)=\frac{K_{1}}{1-K_{1}+\tau s},
$$

where

$$
\begin{aligned}
& K_{1}=\frac{a_{2}^{2}}{\sqrt{a_{1}^{2} \alpha^{2}+a_{2}^{2}}\left(a_{1} \alpha+\sqrt{a_{1}^{2} \alpha^{2}+a_{2}^{2}}\right)}, \\
& \tau=\frac{a_{1}}{\sqrt{a_{1}^{2} \alpha^{2}+a_{2}^{2}}} .
\end{aligned}
$$

Simulation modelling was done for the extrapolator parameters described above.

\section{Simulation modelling}

The described solution simulation modeling was carried out in the MatLab. Channel parameters are: channel bandwidth is equal $8 \mathrm{MHz}$, OFDM subcarriers number - 16. The channel is modeled as a medium with additive white Gaussian noise (AWGN), which power determines the signal-to-noise ratio at the reception at $22 \mathrm{~dB}$. Multipath propagation and radio blackout aren't considered in the model. These baselines and assumptions are correct when the method proposed is being used for the satellite communication channel or line-of-sight channel between pilotless vehicle and terrestrial management and its control center. QPSK is used for quadrature modulation. Earth remote sensing system picture is used as the source of information. Picture size is $512 \times 512$ pixels, image format is BMP without compression, color mode is grayscale. The source picture and its histogram are given in Fig.3
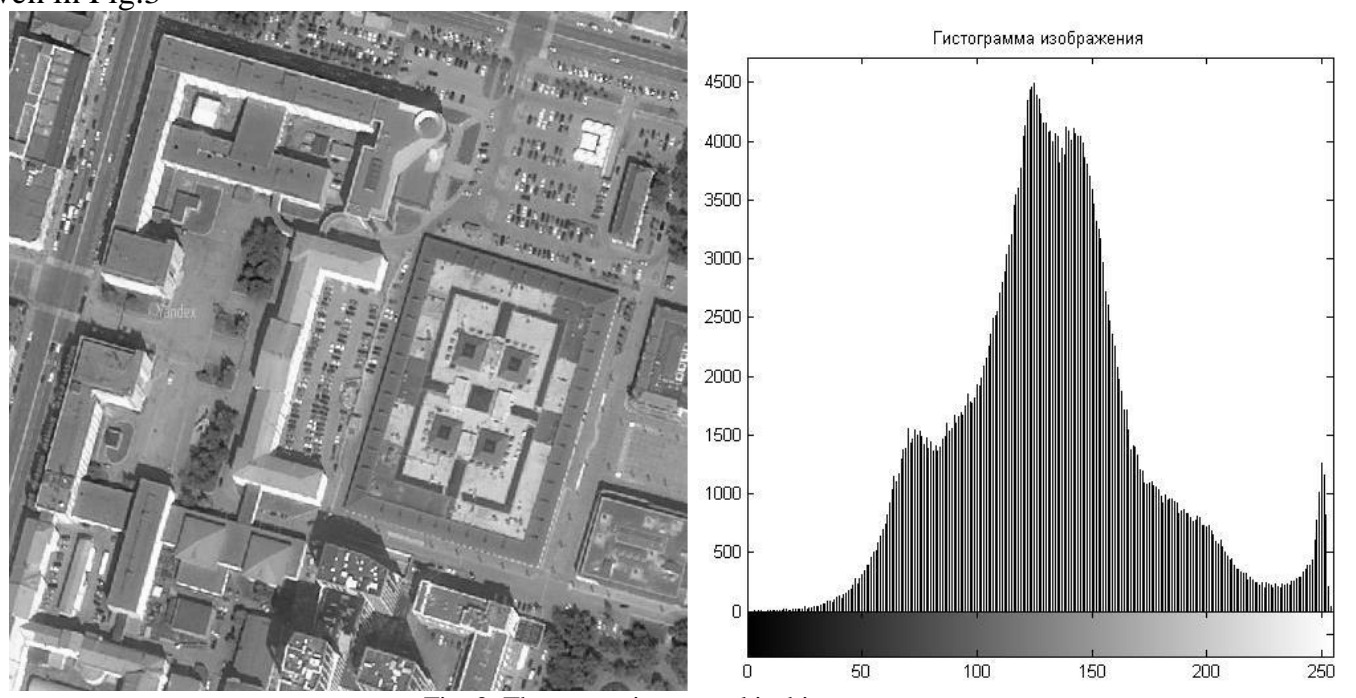

Fig. 3. The source image and its histogram.

The graphic file is divided into $32 \times 32$ pixel fragments, then each of the resulting 256 fragments is being converted to a binary format, after which the QPSK symbols are generated. These symbols are subjected to inverse fast Fourier transform, which allows to obtain the first type of band-pass signal (signal 1). The signal generated is being convoluted with the extrapolator impulse respons, the difference between the original signal and its extrapolated value is calculated, thus, a second type of band signal is generated - a signal compressed in a differential scheme (signal 2). In-phase and quadrature signal shapes before upconvertion for one of the fragments are given in Fig.4. Signal 1 and signal 2 average powers are calculated after that. Third type of signal (signal 3) is being formed with decreasing signal 1 amlitude until this signal power be equal signal 2 power. Transmittion along the AWGN channel is simulated after that. The received band signal is being transformed usin fast Fourier 
Image Processing, Geoinformation Technology and Information Security / G.S. Voronkov, I.V. Kuznetsov, A.Kh. Sultanov

transform. Since 3 variants of the band signal were previously obtained, it is possible to compare QPSK signal constellations obtained by processing different band signals and to estimate the symbol error. The received signal constellations for one of the fragments transmission case are shown in Fig. 5, from left to right: a signal constellation for signal 1, signal 2 and signal 3.
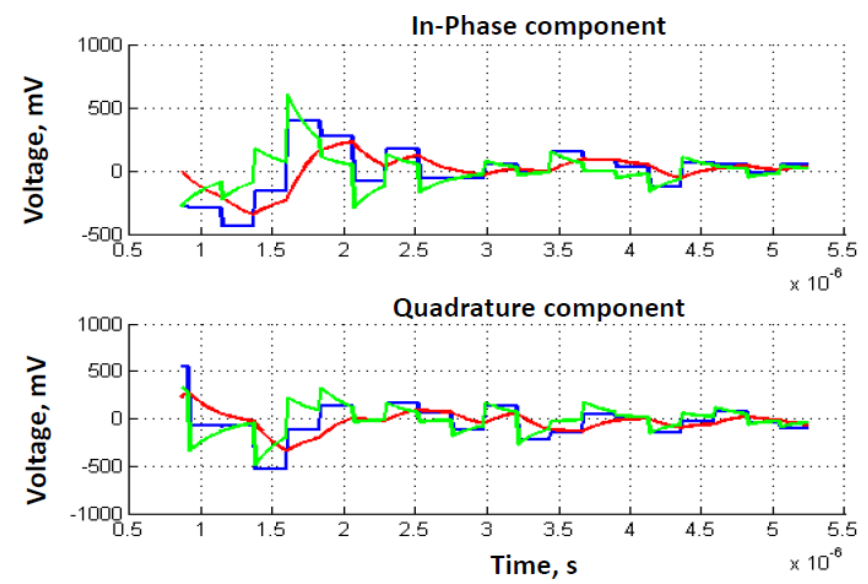

Fig. 4. Signal shapes before upconvertion. Blue - uncompressed signal; Red - extrapolated signal; Green - their difference.
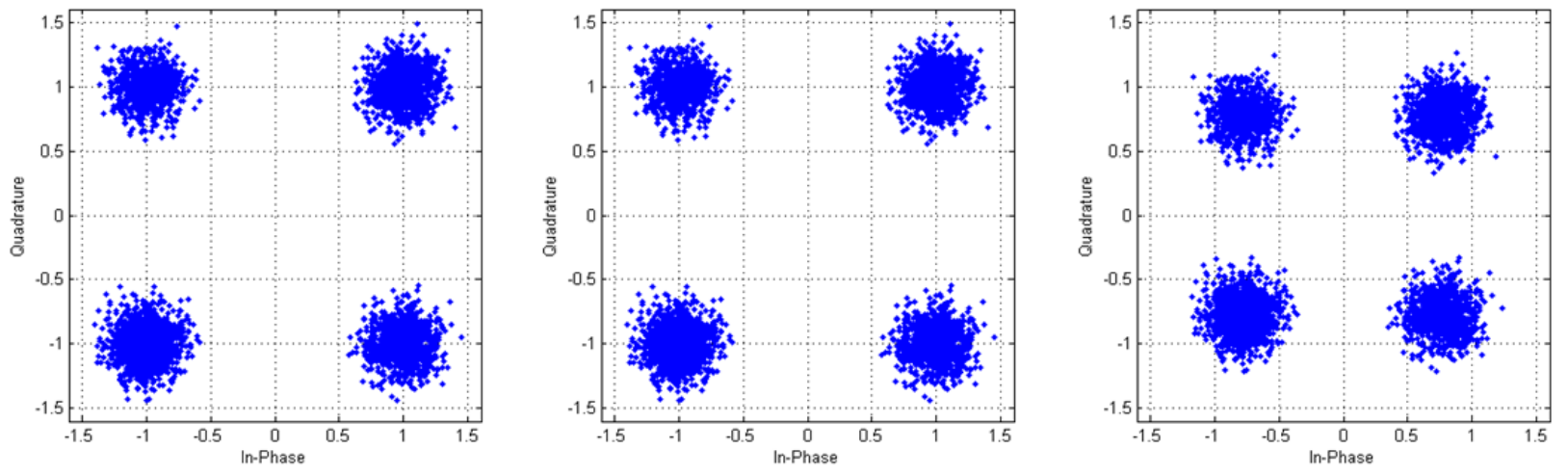

Fig. 5. Received signal constellations.

Since the original image was divided into 256 fragments, there were obtained 256 power gain values for the differential transformation using as a result of modeling. The histogram of compression levels is shown in Fig. 6. The horizontal axis shows the compression ratios, $\mathrm{dB}$, vertical axis - the number of information parcels.

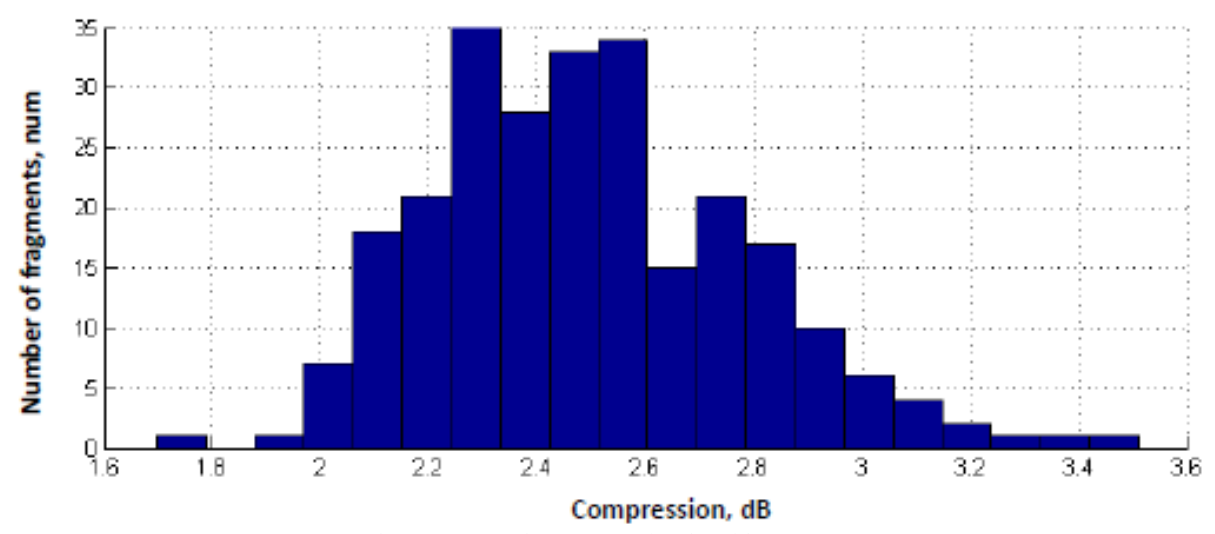

Fig. 6. Dynamic range reduction histogram.

According to the results of the simulation, the differential conversion made it possible to reduce the power of the band signal by $2.49 \mathrm{~dB}$ relative to the original signal. A corresponding reduction in transmitting power without extrapolation leads to an increase in the symbol error. For the uncompressed signal and for the signal subjected to differential transformation, the average symbol error ratio was about $0.000947 \mathrm{~s}^{-1}$, while for the signal with a reduced power the average symbol error ratio was about $0.0638 \mathrm{~s}^{-1}$. The result can also be interpreted in a different way: the use of differential transformation makes it possible to reduce the required signal-to-noise ratio at reception by an average of $2.49 \mathrm{~dB}$ without degrading the communication quality.

\section{Conclusion}

The computational model of OFDM signal differential transformation of based on its extrapolation is presented in the paper. Proposed solution simulation for a short-range radio channel with AWGN without signal multipath propagation and radio 
Image Processing, Geoinformation Technology and Information Security / G.S. Voronkov, I.V. Kuznetsov, A.Kh. Sultanov

blackout is made. The high value of the signal-to-noise ratio given in the experiment is explained by the absence of noise-proof coding in the simulation program. The results of the simulation confirm that the proposed method of differential transformation allows to reduce the amplitude and reduce the average power of the band signal without impairing the noise immunity of the system. Thus, the proposed OFDM signal generation scheme allows to increase the communication system energy efficiency without degrading communication quality.

\section{Acknowledgment}

This work is supported by the Ministry of Education and Science of Russian Federation under the Basic part of the State assignment for higher education organizations 8.5701.2017/BCh.

\section{Reference}

[1] LTE for UMTS: OFDMA and SC-FDMA Based Radio Access. Edited by Harri Holma and Antti Toskala. John Wiley \& Sons Ltd, 2009.

[2] Fundamentals of 5G Mobile Networks. Edited by Jonathan Rodriguez. John Wiley \& Sons Ltd, 2015.

[3] ITU-R M.2047-0. Detailed specifications of the satellite radio interfaces of International Mobile Telecommunications-Advanced (IMT-Advanced).

[4] Gromakov JuA. Mobile radio standards and systems. ECO-TRENDS. Moscow, 1998. (in Russian)

[5] GSM, GPRS and EDGE performance. Evolution Towards 3G/UMTS. Edited by Timo Halonen, Javier Romero, Juan Melero. John Wiley \& Sons Ltd, 2003.

[6] Filatov PE. Increasing the energy-deficient multi-channel communication systems efficiency based on coordinated signal conversion. Applied electrodynamics, photonics and living systems -2016. International Scientific and Technical Conference, 2016; 143-148. (in Russian)

[7] Markiewicz Tomasz G. An Energy Efficient QAM Modulation with Multidimensional Signal Constellation. International Journal of Electronics and Telecommunications 2016; 62(2): 159-165. DOI: 0.1515/eletel-2016-0022.

[8] Kuznetsov IV, Voronkov GS, Sultanov AKh, Antonov VV. Differential OFDM-converter for energy deficient communication system based on coordinated signal predictor design. Radioengineering 2016; 12: 59-63. 